\title{
Customizing WLAN Access Point's Indoor Coverage and Throughput with Corner Reflector
}

\author{
Omar Abdul Aziz ${ }^{1 *}$, Jafri Din ${ }^{1}$, Rozeha A. Rashid ${ }^{2}$, Mohd. Adib Sarijari ${ }^{2}$ and Nur Aqilah Yusri ${ }^{2}$ \\ ${ }^{1}$ Wireless Communication Centre, Universiti Teknologi Malaysia, 81310 UTM Skudai, Johor, Malaysia. \\ ${ }^{2}$ School of Electrical Engineering, Faculty of Engineering, Universiti Teknologi Malaysia.
}

"Corresponding author: omar@utm.my, Tel: +607-5536093

\begin{abstract}
Careful control of indoor wireless coverage is crucial to ensure better signal reception at user-end, mitigate interference to other adjacent wireless systems plus reduce possibility of signal reception by unintended users. Existing solutions are either costly or difficult to configure. This paper presents empirical IEEE802.11 indoor coverage and Transmission Control Protocol (TCP) throughput analysis for WLAN Access Point with corner reflector. The integration of two corner reflector configurations to Access Point was shown to successfully weaken signals by up to $19 \mathrm{~dB}$ and $100 \%$ of TCP throughput while strengthening signals by up to $6 \mathrm{~dB}$ and $40 \%$ of TCP throughput for respective intended regions. The results demonstrate potential application of corner reflector to customize indoor coverage and regulate reliable internet connectivity of Access Point.
\end{abstract}

Keywords: TCP throughput, RSSI, wireless signal strength, Access Point indoor coverage, corner reflector

Article History: received 14 March 2020; accepted 15 April 2020; published 29 August 2020.

(C) 2020 Penerbit UTM Press. All rights reserved

\section{INTRODUCTION}

Current and emergence technologies heavily depend on wireless communication and internet connectivity. The advancement of wireless communication has allowed its application for numerous human activities. The development of Internet of Things (IoT) for example, allows physical objects to be linked wirelessly inside cyber space via electrical components that aid communication between those physical objects [1]. Wireless Local Area Networks (WLANs) provide an alternative link between internet source to users within a specific geographical locality, where physical links are costly to be considered. WLANs operate commonly in an indoor setting. The primary WLAN standards are IEEE 802.11 , or widely known as Wi-Fi [2]. One of the mostly used Wi-Fi system, the IEEE $802.11 \mathrm{~g}$ system, could conventionally be set up to permit information transmission up to $54 \mathrm{Mbps}$.

Transmission control protocol (TCP) make up more than $80 \%$ of the entire Internet's traffic. Forecasting TCP's throughput is therefore essential to improve our understanding of WLAN system's capacity that use TCP to access the internet. In TCP, data packet losses trigger congestion control algorithm to retransmission these lost packets while reducing the sending rate [3]. Nevertheless, evaluating the performance of WLANs is a tremendously difficult task due to variations in interference scenarios as well as the complexities of wireless signal propagation. The physical layer of the Wi-Fi standards stipulates various communication data rates that are dependent on the quality of wireless communication link, or signal-to-noise ratio (SNR) [4]. Generally, indoor signal propagation is more difficult to predict as compared to outdoor signal propagation. This is because signal is affected by more factors, namely building materials, movement of human and machines, walls and obstacles such as furniture. signal wave would experience more scattering, reflection, diffraction and even absorption by building materials. [57].

To regulate wireless signal propagation as per the intended use, several research works have demonstrated better control through smart walls that are made of special materials, but require infrastructure-level renovations [8]. Research work in [9] propose placing several metal plates that may enhance wireless performance but involve a considerable environment changes. A less expensive solution includes incorporating custom-made reflectors to Wi-Fi's Access Point (A.P.).

The proposed reflectors solution presented in [10] however functions with complex reflectors design that are fixed for certain coverage. The corner reflector is an easyto-design reflector that could be easily incorporated with current Wi-Fi's A.P. [11]. Due to the nature of the corner reflector structure, a change in propagation coverage could be easily achieved through position-change of the reflector relative to the A.P. This paper intends to present indoor coverage as well as signal throughput inspections of commercial A.P. with corner reflector through empirical analysis.

\section{MEASUREMENT SETUP}

\subsection{Location}

We conducted measurement campaigns within the ground floor of Wireless Communication Centre (WCC), Universiti Teknologi Malaysia building block. The 
workspace scenario is a $30 \mathrm{~m} \times 21 \mathrm{~m}$ floor area with different room layouts as in Fig. 1. that consists of (a) a spacious indoor lobby area that connects several research laboratory spaces and office rooms. The A.P. is located within this lobby area. 40 measurement points were designated within the lobby with each point being a lineof-sight (LOS) scenario. (b) Non-LOS region 1 that is separated from the lobby by a brick wall. There were 8 designated measurement points within this space. (c) NonLOS region 2 with 12 designated measurement points. This space is separated from the lobby by a dry wall with glass windows. Cumulatively, there were 60 measurement points, represented by the red / blue circle dots in Figure 1.

Received Signal Strength Indicator (RSSI) measurement campaign were conducted twice at different time in order to observe stability of recorded data. At 12 out of the 60 measurement points (represented by the red circle dots in Figure 1), dowstream TCP throughputs were recorded up to four times and averaged during each measurement campaign. Location 1 to 8 were expected to experience drops of RSSI, hence drops of throughput, when corner reflector was applied. On the other hand, location 9 to 10 were where RSSI were expected to increase, hence the throughput as well. Measurement campaign were conducted during working hours with sporadic moving building occupants.

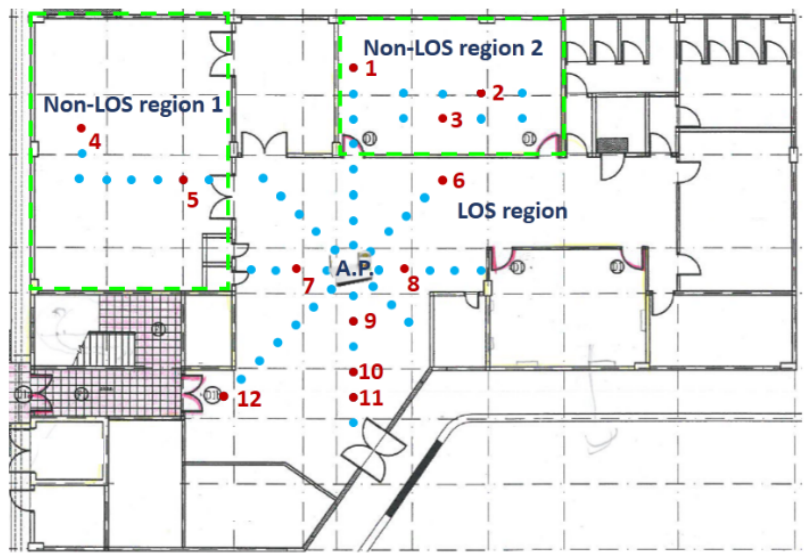

Figure 1. Layout of measurement site for the RSSI and TCP throughput measurement campaigns.

\subsection{Equipment and Tools}

TP-link TL-WR941HP (IEEE 802.11g) was used as A.P. in the measurement campaign. The TL-WR941HP comes with three external $9 \mathrm{dBi}$ omnidirectional antennas operating at $2.4 \mathrm{GHz}$ frequency band. It is worth noting that for this measurement campaign, only one antenna was fixed to the A.P. in order to easily applied the corner antenna as in Figure 2. Impact of applying all three antennas and suitable corner reflector configuration is not covered in this paper.

The A.P. was configured to transmit at $\sim 30 \mathrm{~mW}$. To minimize interference from external A.P.s, channel usage analysis was performed and the A.P. was set to operate on the least used channel (channel 6 for the aforementioned environment). The A.P.'s antenna was positioned $2 \mathrm{~m}$ form the ground, whereas the laptop used as user equipment (UE) was positioned $1.4 \mathrm{~m}$ from the ground.

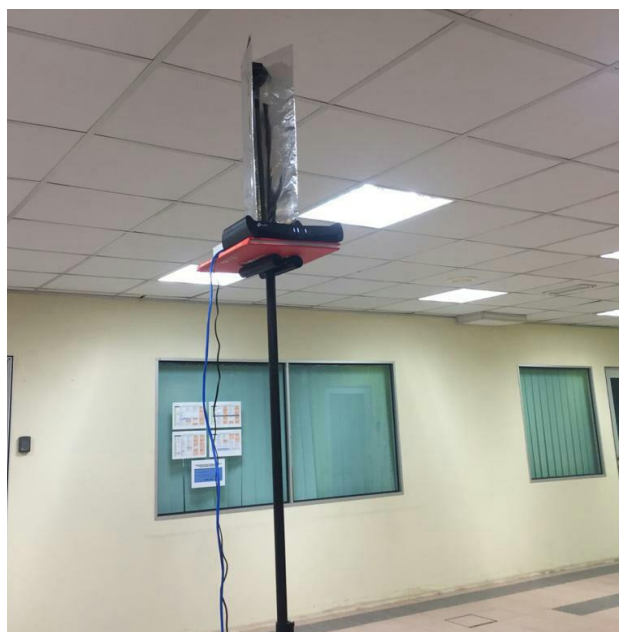

Figure 2. TL-WR941HP with a 30॰ corner reflector configuration.

In order to capture the intended data, modern software tools [12] were installed on a laptop that operated as UE. Acrylic WiFi Scanner and Totusoft's LAN Speed Test (LST) software tools were used to record RSSI and TCP downstream throughput respectively. For throughput data, another laptop with installed LST server software tool was used as a server and connected via RJ45 ethernet cable to the A.P. Tested data packet size was varied with minimum size of $50 \mathrm{~KB}$ and maximum size of $100 \mathrm{~KB}$.

\subsection{Corner Reflector Design}

A corner reflector was developed using heavy-duty aluminum film on a hard board. Aluminum film was selected based on its easily shaped nature, inexpensive, and ability to strongly reflect signal wave as demonstrated in [10]. It has been experimentally observed that a number of rule of thumbs can be used as a guideline when designing an optimized corner reflector antenna. The corner reflector's aperture is commonly made between one and two wavelengths $\left(\lambda<\mathrm{D}_{\mathrm{L}}<2 \lambda\right)$ of the operating frequency. The length of feed to vertex, $\mathrm{S}$, is usually made to be $\lambda / 3<$ $\mathrm{S}<2 \lambda / 3$. The height of the reflector is usually taken to be 1.2 to 1.5 times greater than the total length of the feed element in order to mitigate radiation to the rear region.

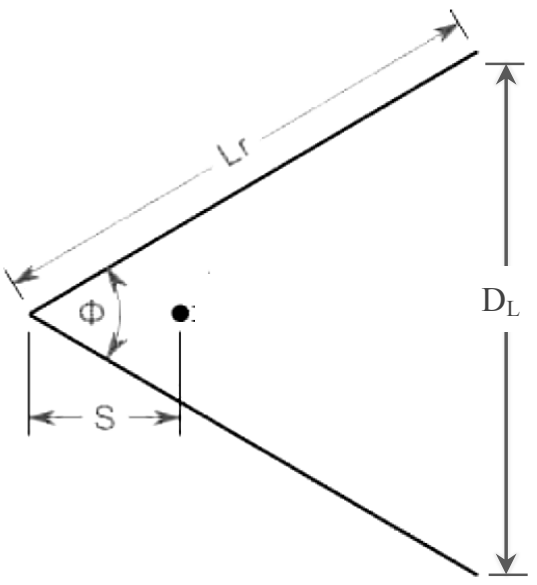

Figure 3. Corner reflector dimension specifications [13]. 
The angle of the reflector, $\theta$, is commonly made to be $\theta$ $=\pi / n$ with $n$ being an integer for proper computation of expected radiation field strength. In most applications, the angle chosen for corner reflector is $90^{\circ}$. Smaller angle reflectors may exhibit better directional characteristics, with consideration of suitable fitting of the feeding element [13]. For this corner reflector dimensions, works in [14] was referred for suitable reflector design as presented in Table 1. The corner reflector's opening angle, $\theta$, was varied to be $90^{\circ}$ and $30^{\circ}$. The corner reflector height was set to be 1.2 times the antenna's length as per the reference, which is $\sim 40 \mathrm{~cm}$. The A.P.'s omnidirectional antenna functions as the feed to the reflector.

Table 1. Corner reflector parameter [13]

\begin{tabular}{|l|l|l|}
\hline No. & \multicolumn{1}{|c|}{ Parameter } & \multicolumn{1}{c|}{ Value } \\
\hline 1. & Operating frequency & $2.4 \mathrm{GHz}$ \\
\hline 2. & $\begin{array}{l}\text { Feed to Vertex Distance } \\
(\mathrm{S})\end{array}$ & $0.67 \lambda$ \\
\hline 3. & Angle $(\theta)$ & $90^{\circ}$ and $30^{\circ}$ \\
\hline 4. & Length $(\mathrm{Lr})$ & $0.75 \lambda$ \\
\hline
\end{tabular}

\section{RESULTS AND DISCUSSIONS}

Figure 4 presents the recorded RSSI for the two measurement campaigns carried out. The figures show that both measurement campaigns recorded rather similar RSSI trends against separation distance between A.P. and UE.

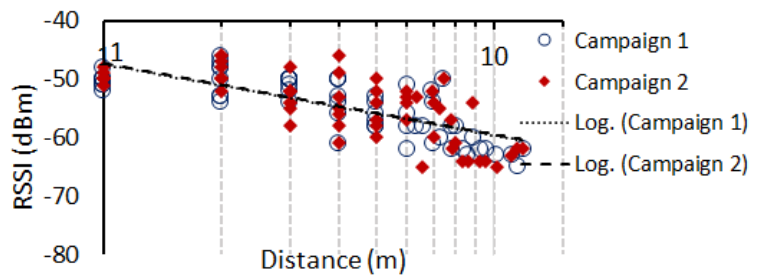

(a)

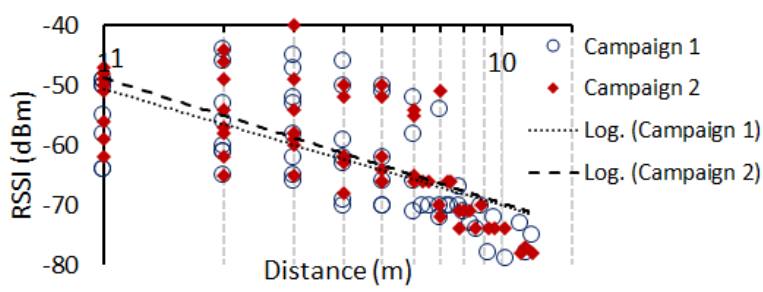

(b)

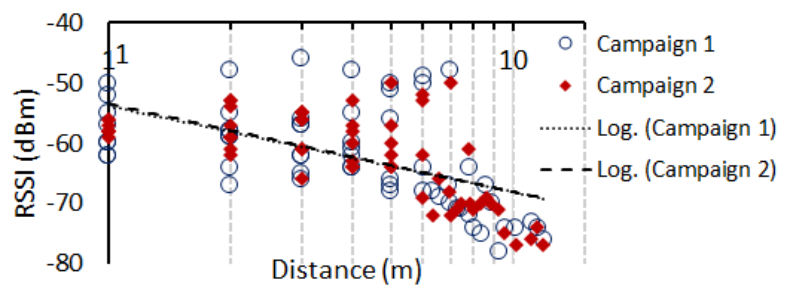

(c)

Figure 4. Recorded RSSI at all 60 measurement points for A.P. (a) Without corner reflector (b) With $90^{\circ}$ corner reflector and (c) With 30॰ corner reflector.
Standard deviation of recorded data between the two campaigns were $2.3 \mathrm{~dB}, 1.8 \mathrm{~dB}$ and $2.5 \mathrm{~dB}$ for A.P. without reflector, with $90^{\circ}$ and $30^{\circ}$ reflector respectively. Some observations from fig. 4 include; variation of RSSI values at a specific distance becomes greater with the application of corner reflector given that several of the locations experience increase in RSSI while at other locations of similar distance experience decrease in RSSI.

Figure 5. presents the coverage of A.P. based on plotted average RSSI recorded from the two measurement campaign, at each investigated setting. Generally, the application of corner reflector was shown to clearly reduce and improve RSSI at the back and front regions of the corner reflector.

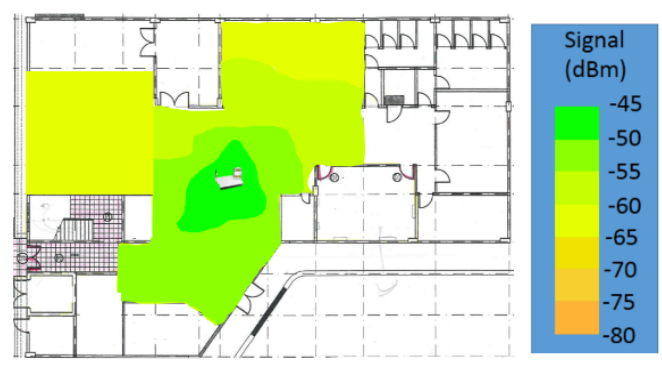

(a)

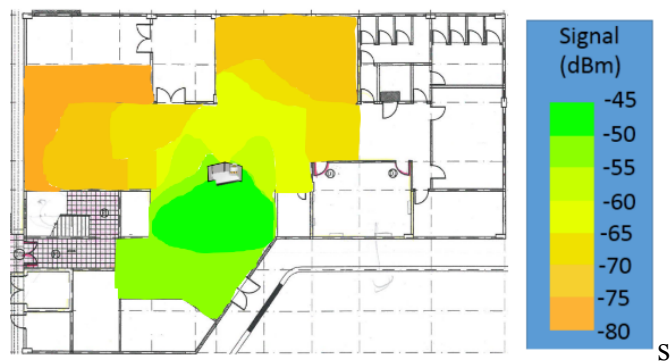

(b)

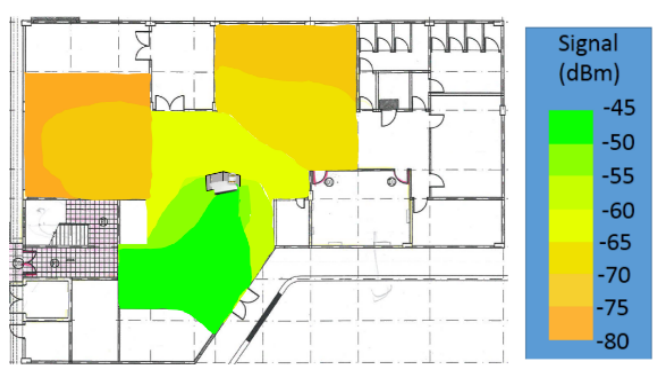

(c)

Figure 5. Indoor coverage for A.P. (a) Without corner reflector (b) With 90。 corner reflector and (c) With 30。 corner reflector.

With the design of corner reflector used, the $30^{\circ}$ corner reflector configuration was shown to have reduced backlobes, narrower beamwidth plus greater gain when compared to the $90 \circ$ corner reflector. Figure 6 presents the SNR at the 12 selected points where throughput data was recorded. Conforming to findings in [10], signal strengthening demonstrated smaller change (up to $6 \mathrm{~dB}$ ) when compared to signal weakening (up to $19 \mathrm{~dB}$ ) with the application of corner reflector investigated. 


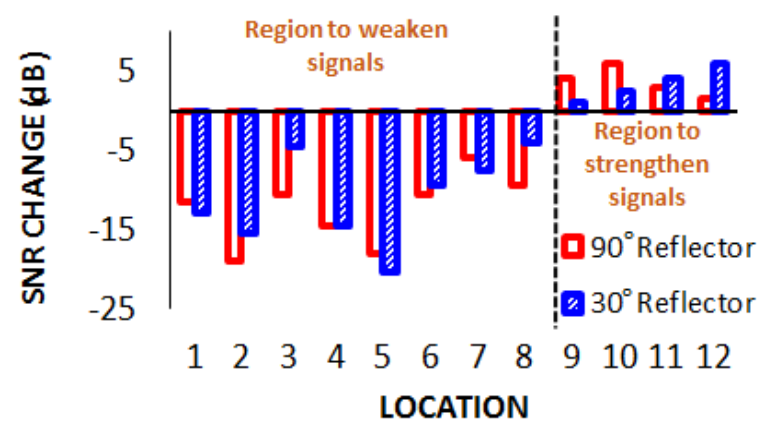

Figure 6. SNR change with corner reflector at the A.P.

Figure 7 (a) and (b) present average TCP downstream throughput change when corner reflector is applied at the 12 selected points in $\mathrm{Mb} / \mathrm{s}$ and corresponding percentage change. An increase or decrease in SNR correspond directly to an increase or decrease in throughput at all settings except for location 12 with $90^{\circ}$ corner reflector, where a drop of $0.1 \mathrm{Mbps}$ was recorded, thus showing the dynamic involve in throughput evaluation in this investigation. Average throughput improvement for region with signals strengthened was about $18 \%$ and $22 \%$ for the $90^{\circ}$ and $30^{\circ}$ reflector respectively. On the other hand, the average throughput drop for region with weakened signals are $69 \%$ and $86 \%$ for the $90 \circ$ and $30^{\circ}$ reflector respectively. It should be noted, that several of the locations with weakened signals recorded throughput decrement of $100 \%$ from when no reflector was applied.

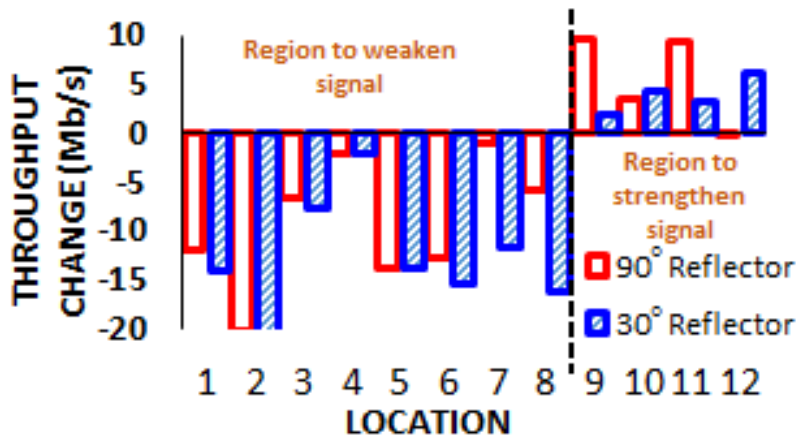

(a)

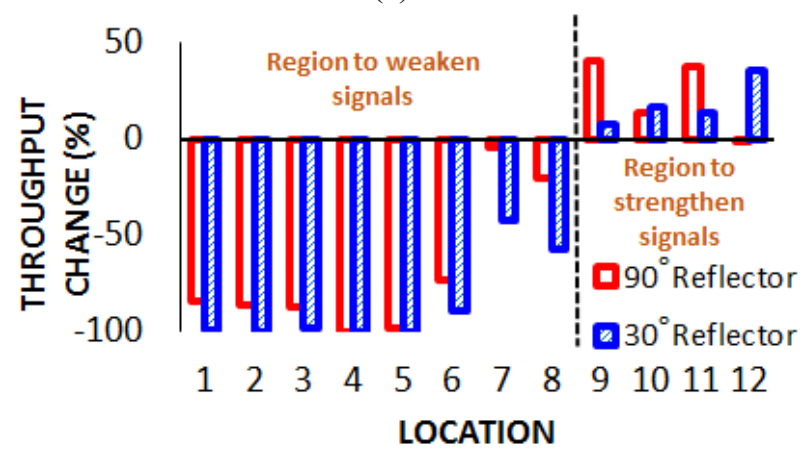

(b)

Figure 7. Throughput change with corner reflector at the A.P. in (a) Mb/s and (b) percentage.

\section{CONCLUSION}

This paper has presented empirical investigation of coverage and throughput evaluation for two corner reflector configurations. The findings demonstrate that corner reflector aperture may also influence performance of A.P. The percentage throughput change was in fact better than the complex design presented in [10]. Changes in corner reflector aperture, and orientation could be easily applied to A.P. in order to customize coverage, hence SNR plus throughput for the A.P. in operation.

\section{ACKNOWLEDGMENT}

The authors would like to express gratitude to the Malaysian Ministry of Education for the provided WCCUTM Higher Institution Centre of Excellence (HICoE) phase 2 research grant Vot. No. RJ130000.7851.4J409 entitled Characterizing Shielding Impact on the Privacy of Machine-machine Communication in Indoor-Outdoor scenario from which this research was carried out.

\section{REFERENCES}

[1] 5G Spectrum Recommendation, 4G Americas, America, August 2015.

[2] Kalpana, P. Jeevana Pravallika, B. Ratna Kesava Reddy, S. S. S. Aravind, S. Dinesh Kumar, "Design And Simulation of Wire and Planar Antenna using 4NEC2," 2016.

[3] Moltchanov, D., 2012, A study of TCP performance in wireless environment using fixed-point approximation.

[4] Oghogho I. (2018a) Throughput dependence on SNR in IEEE 802.11 WLAN systems. In Ed. Mehdi Khosrow-Pour, Encyclopedia of Information, Science and Technology, Fourth Edition. IGI, Global USA.pp 6618-6629.

[5] J. S. Seybold, Introduction to $R F$ Propagation, Hoboken, New Jersey, Canada: John Wiley \& Sons, Inc., 2005

[6] T. Rappaport, Wireless Communications: Principle and Practice. Englewood Cliffs, NJ, USA: Prentice Hall, 1996.

[7] W. Stallings, Wireless Communications and Networks Second Edition, Upper Saddle River, NJ, USA: Prentice Hall, 2005.

[8] Dupre, M., et al. Recycling radio waves with smart walls. In International Conference on Metamaterials, Photonic Crystals and Plasmonics (2015).

[9] Han, S., and Shin, K. G. Enhancing Wireless Performance Using Reflectors. In Proc. of INFOCOM (2017).

[10] Xiong. X.. Chan. J.. Yu. E.. Kumari. N.. Sani. A. A.. Zheng. C.. \& Zhou. X. (2017. November). Customizing indoor wireless coverage via $3 \mathrm{~d}-$ fahricated reflectors. In Proceedings of the 4th ACM International Conference on Sustems for EnergyEfficient Built Environments (p. 8). ACM.

[11] Dimousios. T. D.. Mitilineos. S. A.. Panagiotou. S. C.. \& Cansalis. C. N. (2011). Design of a corner-reflector reactivelv controlled antenna for maximum directivitv and multiple beam forming at $2.4 \mathrm{GHz}$. IEEE Transactions on Antennas and Propagation, 59(4), 1132-1139.

[12] Male, S., Vatti, R., Meshram, T., \& Bhanap, O. (2018). Wireless Network Signal Strength Measurement Tools: A Survey. S-RATE International Journal of Research in Application Technologies, $1(1), 5-9$.

[13] Balanis. C. A. (2016). Antenna theory: analysis and design. John wiley \& sons.

[14] Sam. J. K. A. (2014). Corner Reflector Antenna Design for Interference Mitigation between FM Broadcasting and Aeronautical Ground to Air Communication Radios. 